\title{
Staircase functions, spectral rigidity, and a rule for quantizing chaos
}

\author{
R. Aurich and F. Steiner \\ II. Institut für Theoretische Physik, Universität Hamburg, Luruper Chaussee 149, \\ 2000 Hamburg 50, Federal Republic of Germany \\ (Received 2 August 1991)
}

\begin{abstract}
Considering the Selberg trace formula as an exact version of Gutzwiller's semiclassical periodic-orbit theory in the case of the free motion on compact Riemann surfaces with constant negative curvature (Hadamard-Gutzwiller model), we study two complementary basic problems in quantum chaology: the computation of the classical staircase $N(l)$, the number of periodic orbits with length shorter than $l$, in terms of the quantal energy spectrum $\left\{E_{n}\right\}$; the computation of the spectral staircase $\mathcal{N}(E)$, the number of quantal energies below the energy $E$, in terms of the length spectrum $\left\{l_{n}\right\}$ of the classical periodic orbits. A formulation of the periodic-orbit theory is presented that is intrinsically unsmoothed, but for which an effective smoothing arises from the limited "input data," i.e., from the limited knowledge of the periodic orbits in the case of $\mathcal{N}(E)$ and the limited knowledge of quantal energies in the case of $N(l)$. Based on the periodic-orbit formula for $\mathcal{N}(E)$, we propose a rule for quantizing chaos, which simply states that the quantal energies are determined by the zeros of the function $\xi_{1}(E)=\cos [\pi \mathcal{N}(E)]$. The formulas for $N(l)$ and $\mathcal{N}(E)$ as well as the quantization condition are tested numerically. Furthermore, it is shown that the staircase $\mathcal{N}(E)$ computed from the length spectrum yields (up to a constant) a good description of the spectral rigidity $\Delta_{3}(L)$, and thus we are able to compute a statistical property of the quantal energy spectrum of a chaotic system from classical periodic orbits.
\end{abstract}

PACS number(s): 05.45. $+\mathrm{b}, 03.65 .-\mathrm{w}$

\section{INTRODUCTION}

In this paper we discuss as an application of Gutzwiller's semiclassical periodic-orbit theory $[1,2]$ the computation of discontinuous staircase functions and of the spectral rigidity. To this end we employ an unsmoothed version of the periodic-orbit theory. Usual$1 y$, the convergence of the periodic-orbit sums is enforced by a suitable smoothing procedure. In the formulation given here no explicit smoothing is needed, since the smoothing of the periodic-orbit theory arises automatically from the limited knowledge of the "input data."

The periodic-orbit theory is applied to the HadamardGutzwiller model, a chaotic system which consists of a point particle sliding freely on a compact Riemann surface of genus $g=2$ and with constant negative curvature. In this case Gutzwiller's periodic-orbit theory [1] is exact, as Gutzwiller recognized [3] by comparing his semiclassical theory with the Selberg trace formula [4]. This system is a conservative $K$ system and possesses only a discrete quantal energy spectrum $\left\{E_{n}\right\}$ $\left(0=E_{0}<E_{1} \leq E_{2} \leq \cdots\right)$. An introduction to this strongly chaotic model can be found in $[2,5]$ or in some of our previous papers [6-8].

We discuss two complementary basic problems of quantum chaology. On the one hand, the number $N(l)$ of primitive periodic orbits with lengths $l_{n}$ shorter than $l$ is computed from the quantal energy spectrum $\left\{E_{n}\right\}$. According to Huber's law [9], this number is exponentially growing, $N(l) \sim e^{l} / l$ for $l \rightarrow \infty$, as it is typical for chaotic systems. On the other hand, the periodic-orbit theory allows the computation of the number $\mathcal{N}(E)$ of quantal energies $E_{n}$ less than $E$ from the length spectrum $\left\{l_{n}\right\}$ of the classical periodic orbits. In our numerical applications we use the regular octagon, for which the length spectrum $\left\{l_{n}\right\}$ is completely known [10] up to $n=1500\left(l_{1500}=18.092 . ..\right)$ and an asymmetric octagon, for which the length spectrum is nearly completely known up to $l=12$. Here the number $n$ counts only the periodic orbits of different lengths. Indeed, there are more than 4,000000 periodic orbits up to $n=1500$ in the case of the regular octagon.

Based on the periodic-orbit formula for $\mathcal{N}(E)$ we formulate a quantization rule for chaotic systems. A successful test of this quantization condition is performed in the case of the two octagons. Finally, the spectral staircase is used to compute the spectral rigidity $\Delta_{3}(L)$ from a knowledge of the classical periodic orbits.

The starting point is Selberg's trace formula [4]

$$
\begin{aligned}
\sum_{\left\{p_{n}\right\}} h\left(p_{n}\right)= & \frac{A(\mathcal{F})}{4 \pi} \int_{-\infty}^{\infty} d p p \tanh (\pi p) h(p) \\
& +\sum_{\left\{l_{n}\right\}} \sum_{k=1}^{\infty} \frac{l_{n}}{2 \sinh \left(k l_{n} / 2\right)} g\left(k l_{n}\right),
\end{aligned}
$$

where $A$ is the area, $p_{n}=\sqrt{E_{n}-\frac{1}{4}}$ are the momenta, and $h(p)$ is a (nearly) arbitrary even function that is holomorphic in the strip $|\operatorname{Im} p| \leq \frac{1}{2}+\epsilon, \epsilon>0$, and vanishes asymptotically for $|p| \rightarrow \infty$ faster than $1 / p^{2}$. The lefthand side (lhs) of (1) can be viewed as of purely quantum-mechanical origin, whereas the right-hand side (rhs) is purely classical nature involving the so-called 
zero-length term proportional to the area of the octagons, $A(\mathcal{F})=4 \pi$, and the periodic-orbit sum over the classical length spectrum $\left\{l_{n}\right\}$. The Fourier transform of $h(p)$ is denoted by $g(x)$,

$$
g(x)=\frac{1}{\pi} \int_{0}^{\infty} d p \cos (p x) h(p)
$$

\section{THE CLASSICAL STAIRCASE $\boldsymbol{N}(l)$}

In [7] we discussed the trace of the cosine-modulated heat kernel, which is obtained from the Selberg trace formula (1) by choosing

$$
h(p)=\cos (p L) e^{-E t}, \quad E=\frac{1}{4}+p^{2} .
$$

The resulting sum rule reads

$$
\begin{aligned}
\cosh \frac{L}{2}+\sum_{n=1}^{\infty} \cos \left(p_{n} L\right) e^{-E_{n} t}= & 2 e^{-t / 4} \int_{0}^{\infty} d p p \tanh (\pi p) \cos (p L) e^{-p^{2} t} \\
& +\frac{e^{-t / 4}}{8 \sqrt{\pi t}} \sum_{\left\{l_{n}\right\}} \sum_{k=1}^{\infty} \frac{l_{n}}{\sinh \left(k l_{n} / 2\right)}\left(e^{-\left(L-k l_{n}\right)^{2} / 4 t}+e^{-\left(L+k l_{n}\right)^{2} / 4 t}\right)
\end{aligned}
$$

which is absolutely convergent for any $t>0$. The first term on the lhs of Eq. (4) is due to the zero ground-state energy $E_{0}=0 \quad\left(p_{0}=i / 2\right)$, while the second term represents a sum over all eigenvalues $E_{n}>\frac{1}{4}$. (The octagons discussed in this paper possess no so-called "small" eigenvalues with $0<E_{n} \leq \frac{1}{4}$.) The last term on the rhs of the sum rule (4) shows at fixed $t>0$ as a function of the real variable $L$ Gaussian peaks of width $\Delta L \sim 2 \sqrt{2 t}$ at the lengths $l_{n}$ of the classical periodic orbits. In [7] we presented an evaluation of (4) for $t=0.01$, resolving the periodic orbits of short lengths. (A preliminary evalua- tion has been presented already in [11].)

Here we are interested in the unsmoothed staircase function $N(l)$, which can be obtained from (4) as follows. Applying the operation

$$
\int_{l_{0}}^{l} d L \frac{4}{L} \sinh \frac{L}{2}, \quad 0<l_{0}<l_{1}<l,
$$

where $l_{1}$ is the length of the shortest periodic orbit, on both sides of Eq. (4) and performing the limit $t \rightarrow 0+$, in which the Gaussian peaks degenerate to Dirac $\delta$ functions, yields

$$
\begin{aligned}
\left.\sum_{k=1}^{\kappa(l)} \frac{1}{k} \sum_{n=1}^{\infty} \int_{l_{0}}^{l} d L\left(\frac{k l_{n}}{L}\right) \int \frac{\sinh (L / 2)}{\sinh \left(k l_{n} / 2\right)}\right) \delta\left(L-k l_{n}\right)= & \int_{l_{0}}^{l} d L \frac{4 \sinh (L / 2) \cosh (L / 2)}{L} \\
& +\sum_{n=1}^{\infty} \int_{l_{0}}^{l} d L \frac{4 \sinh (L / 2) \cos \left(p_{n} L\right)}{L} \\
& +\int_{l_{0}}^{l} d L \frac{4 \sinh (L / 2)}{L} \frac{\cosh (L / 2)}{2 \sinh ^{2}(L / 2)},
\end{aligned}
$$

with $\kappa(l) \equiv\left[l / l_{1}\right]$. The lhs of (6) can be rewritten in terms of the classical staircase $N(l)$ :

$$
\begin{aligned}
\sum_{k=1}^{\kappa(l)} \frac{1}{k} \sum_{n=1}^{\infty} \int_{l_{0}}^{l} d L \delta\left(L-k l_{n}\right) & =\sum_{k=1}^{\kappa(l)} \frac{1}{k} \sum_{n=1}^{\infty} \Theta\left(\frac{l}{k}-l_{n}\right) \\
& =\sum_{k=1}^{\kappa(l)} \frac{1}{k} N\left(\frac{l}{k}\right)
\end{aligned}
$$

If the rhs of (6) is denoted by $F(l)$,

$$
\begin{aligned}
& F(l) \equiv \int_{l_{0}}^{l} \frac{d L}{L}\left(e^{L}-e^{-L}\right) \\
& +4 \sum_{n=1}^{\infty} \int_{l_{0}}^{l} \frac{d L}{L} \sinh \frac{L}{2} \cos \left(p_{n} L\right) \\
& \quad+2 \int_{l_{0}}^{l} \frac{d L}{L} \operatorname{coth} \frac{L}{2},
\end{aligned}
$$

we arrive at the basic relation

$$
\sum_{k=1}^{\kappa(l)} \frac{1}{k} N\left(\frac{l}{k}\right)=F(l),
$$

which counts the number of periodic orbits up to the length $l$ including multiple traversals $(k>1)$ weighted by $1 / k$. Equation (9) can be solved for $N(l)$ by using the Möbius inversion formula [12], yielding

$$
N(l)=\sum_{k=1}^{\kappa(l)} \frac{\mu(k)}{k} F\left(\frac{l}{k}\right),
$$

where $\mu(k)$ is the Möbius function $[\mu(1)=1, \mu(2)=-1$, $\mu(3)=-1, \mu(4)=0, \mu(5)=-1, \mu(6)=1, \cdots]$. Thus the classical staircase function $N(l)$ can be expressed exactly by the function $F(l)$, Eq. (8), which in turn is completely determined by the quantal energy spectrum.

Equations (8)-(10) are very similar to the primenumber formulas involving $\pi(x)$ published by Riemann in his famous paper [13] in 1859 and proved by von Man- 
goldt [14] in 1895 , where $\pi(x)$ is the number of prime numbers not exceeding $x$. Riemann obtained for the leading term for $x \rightarrow \infty$ (prime number theorem)

$$
\pi(x) \sim \operatorname{li}(x)
$$

where the logarithmic integral is defined by the principal value integral

$$
\operatorname{li}(x)=\mathrm{P} \int_{0}^{x} \frac{d t}{\ln t}, x>1 .
$$

The prime-number theorem (11) was proved independently by Hadamard [15] and de la Vallée Poussin [16] in 1896. Notice that (11) is equivalent to

$$
\pi(x) \sim \frac{x}{\ln x}, \quad x \rightarrow \infty .
$$

That (11) gives a good approximation, was already noticed empirically by Gauss. Riemann was the first to write down an explicit expression for the remainder. Riemann's formula reads $[13,17]$

$$
\pi(x)=\sum_{k=1}^{[\ln x / \ln 2]} \frac{\mu(k)}{k} f\left(x^{1 / k}\right),
$$

where the function $f(x)$ has the explicit representation

$$
f(x)=\operatorname{li}(x)-\sum_{\left\{\rho_{n}\right\}} \operatorname{li}\left(x^{\rho_{n}}\right)+\int_{x}^{\infty} \frac{d t}{\left(t^{2}-1\right) t \ln t}-\ln 2 .
$$

Here the sum over $\rho_{n}$ runs over all the nontrivial zeros of the Riemann $\xi$ function.

To compare with our relations $(8)-(10)$, we make a change of variables, $x=e^{l}$, which yields

$$
\operatorname{li}\left(e^{l}\right)=\mathrm{P} \int_{-\infty}^{l} \frac{d L}{L} e^{L} \equiv \operatorname{Ei}(l),
$$

and the prime-number theorem now reads

$$
\pi\left(e^{l}\right) \sim \operatorname{Ei}(l) \sim \frac{e^{l}}{l}, \quad l \rightarrow \infty .
$$

Using the definition (16), the function $F(l)$ in Eq. (8) can be rewritten as

$$
F(l)=\operatorname{Ei}(l)+\sum_{\left\{s_{n}\right\}} \operatorname{Ei}\left(s_{n} l\right)+2 \ln l+O(1),
$$

where the sum over $s_{n}$ runs over all pairs of nontrivial zeros of the Selberg $\zeta$ function [4], i.e., $s_{n}=\frac{1}{2} \pm i p_{n}, p_{n}>0$ (also see Sec. VII).

The leading term of Eq. (18) is given by

$$
F(l) \sim \operatorname{Ei}(l), \quad l \rightarrow \infty,
$$

which yields with (10) the asymptotic behavior

$$
N(l) \sim \operatorname{Ei}(l), \quad l \rightarrow \infty .
$$

Equation (20) shows the well-known exponential proliferation of periodic orbits for chaotic systems in general, which was first derived by Huber [9]. [Huber also derived the corrections that are present if there exist small eigenvalues $0<E_{n} \leq \frac{1}{4}$. The result is given by the second term in Eq. (18), where the sum over $s_{n}$ runs over all real values $\frac{1}{2} \leq s_{n}<1, E_{n}=s_{n}\left(1-s_{n}\right)$.] A comparison of Eqs. (20) and (17) shows that Huber's law (20) plays the role of a "generalized prime-number theorem" for the "generalized primes" $p_{\gamma}$ (sometimes called pseudoprimes) defined by $p_{\gamma} \equiv \operatorname{norm}(\gamma)=e^{l} \gamma$, where $\gamma$ denotes a primitive hyperbolic element of the group $\Gamma$ associated with the chosen octagon. Here $\Gamma$ is a discrete subgroup of $\operatorname{PSL}(2, \mathbb{R}), \equiv S L(2, \mathbb{R}) /\{ \pm \mathbb{1}\}$, and $l_{\gamma}$ is the length of the closed geodesic (=periodic orbit) uniquely associated with a given hyperbolic element $\gamma$. [Notice that the numbers $l_{p} \equiv \ln p$, where $p$ runs over all primes, play the role of the lengths of fictitious periodic orbits in the case of $\pi(x)$.]

In Ref. [6] we have presented the first computation of a length spectrum for a compact Riemann surface. A complete enumeration of all periodic orbits not exceeding $l=18.092 .$. has been given in the case of the regular octagon in [10]. In Figs. 2 and 3 of Ref. [10] we have illustrated that Huber's law (20) yields an excellent approximation to the true staircase function $N(l)$ in the range $l_{1} \leq l \leq l_{1500}=18.092 \ldots$. These figures show that $\operatorname{Ei}(l)-N(l)$ has a great number of sign changes, which often characterizes a good approximation. This is in contrast to the approximation (11) in the case of prime numbers, where available tables [17] show that $\operatorname{li}(x)-\pi(x)$ is always positive for $x \leq 4 \times 10^{16}$ and increasing. Indeed, such a behavior is suggested by Eq. (14), which gives $\operatorname{li}(x)-\pi(x) \sim \frac{1}{2} \operatorname{li}(\sqrt{x})$, if $f(x)$ is approximated by the first term in Eq. (15). Although no one so far has found any specific example of $\operatorname{li}(x)-\pi(x)<0$ [17], Littlewood has shown [18] that $\operatorname{li}(x)-\pi(x)$ changes sign infinitely often. (For a discussion of the properties of primes, we refer the reader to Riesel's book [17] and to the lecture by Zagier [19].)

If the Ei function in the second term of Eq. (18) is approximated by its leading term, see Eq. (17), we obtain $\left[\alpha_{n}=\arctan \left(2 p_{n}\right)\right]$

$F(l)=\operatorname{Ei}(l)+2 \frac{e^{l / 2}}{l} \sum_{n=1}^{\infty} \frac{\cos \left(p_{n} l-\alpha_{n}\right)}{\sqrt{E_{n}}}+\cdots$,

and thus from Eq. (10)

$N(l)=\operatorname{Ei}(l)-\frac{e^{l / 2}}{l}+2 \frac{e^{l / 2}}{l} \sum_{n=1}^{\infty} \frac{\cos \left(p_{n} l-\alpha_{n}\right)}{\sqrt{E_{n}}}+\cdots$.

This formula shows explicitly how the fine structure of the length spectrum of the classical periodic orbits is determined by the quantal energies ("inverse quantum chaology"), in the same way as the distribution of primes is determined by the nontrivial Riemann zeros via Eq. (15). If the sum in Eq. (22) is approximated by taking into account all energies with $E_{n} \leq E_{N}$, one should get a resolution of $\Delta l \sim \pi / \sqrt{E_{N}}$, i.e., $\Delta l \sim 0.22$ for $E_{N}=200$. If more and more quantal energies are included in the sum, one expects a kind of Gibbs phenomenon in analogy with the well-known behavior for classical Fourier series. In Ref. [20] a calculation of $\pi(x)$ has been presented by using Riemann's prime-number formulas (14) and (15) 


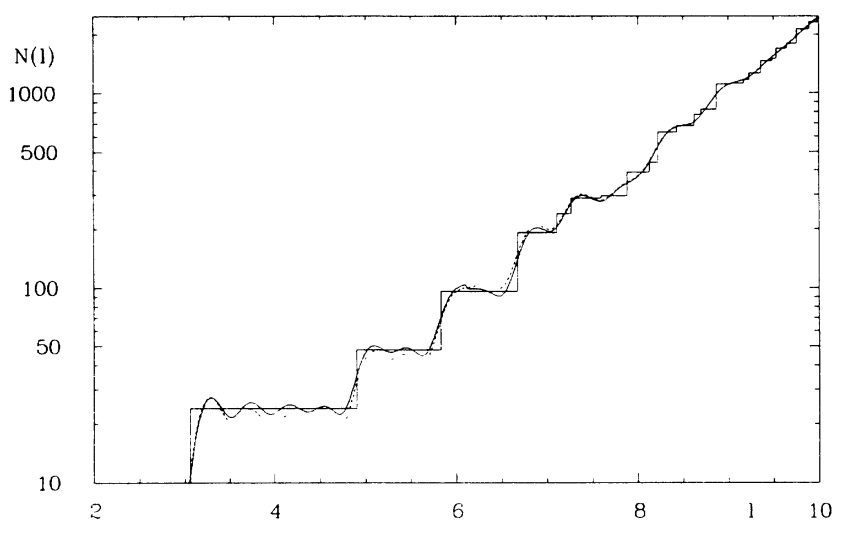

FIG. 1. The classical staircase $N(l)$ is shown for the regular octagon in comparison with the result obtained from (10) using (8) for $l_{0}=1$ and $E_{N}=201.4 \ldots$ The asymptotic formula (22) is represented by the dotted curve.

and taking into account the first 29 Riemann zeros.

In Fig. 1 the exact staircase $N(l)$ of the regular octagon is shown for $l \leq 10$ together with the curve obtained from (10) and (8), where only the first 200 quantal energies has been used, i.e., $E_{N}=201.4 \ldots$. As the lower integration limit, $l_{0}=1$ has been chosen. Remarkably, the result should be independent of the choice of $l_{0}$ as long as $0<l_{0}<l_{1}$ is fulfilled. Numerically one observes a slight dependence on $l_{0}$ because of the truncation of the series over the quantal energies. This truncation smoothes the discontinuities of the true $N(l)$, and if $l_{0}$ is chosen too near the shortest length, the curve lies slightly below the true one. This is obvious, noticing that for $l_{1}<l_{0}<l_{2}$ the first length $l_{1}=3.057 \ldots$ would have been omitted in $N(l)$. Although (10) and (8) are unsmoothed, the restricted knowledge of the quantal energies leads to a smoothing. Thus this smoothing is due only to the limited "input data" and not to an explicitly smoothed formula.

\section{THE SPECTRAL STAIRCASE $\mathcal{N}(E)$}

In [7] we have already obtained a smoothed version for the spectral staircase $\mathcal{N}(E)$ starting with the Gaussian smoothed sum rule. For a given smoothing parameter $\epsilon>0$ we have derived the absolutely convergent relation

$$
\begin{aligned}
& \mathcal{N}_{\epsilon}(E)=\frac{4}{\sqrt{\pi} \epsilon} \int_{0}^{\infty} d p^{\prime \prime} p^{\prime \prime} \tanh \pi p^{\prime \prime} \\
& \quad \times \int_{0}^{p} d p^{\prime} \cosh \frac{2 p^{\prime} p^{\prime \prime}}{\epsilon^{2}} e^{-\left(p^{\prime 2}+p^{\prime \prime 2}\right) / \epsilon^{2}} \\
& +\frac{1}{2 \pi} \sum_{\left\{l_{n}\right\}} \sum_{k=1}^{\infty} \frac{\sin \left(p k l_{n}\right)}{k \sinh \left(k l_{n} / 2\right)} e^{-\left(\epsilon k l_{n} / 2\right)^{2}} .
\end{aligned}
$$

Here we are interested in the limit $\epsilon \rightarrow 0$ because of $\lim _{\epsilon \rightarrow 0} \mathcal{N}_{\epsilon}(E)=\mathcal{N}(E)$. Performing the limit one arrives at $\left(p=\sqrt{E-\frac{1}{4}}>0\right)$

$$
\begin{aligned}
\mathcal{N}(E)= & 2 \int_{0}^{p} d p^{\prime} p^{\prime} \tanh \left(\pi p^{\prime}\right) \\
& +\frac{1}{2 \pi} \sum_{\left\{l_{n}\right\}} \sum_{k=1}^{\infty} \frac{\sin \left(p k l_{n}\right)}{k \sinh \left(k l_{n} / 2\right)} .
\end{aligned}
$$

At first sight the last relation, which is valid in the sense of distributions, seems to be useless for a numerical application, because for $\epsilon=0$ there is no suppression of the exponentially increasing number $N(l)$ of periodic orbits with increasing length $l$, and thus one expects numerical instabilities.

As in [21] let us assume that the length spectrum $\left\{l_{n}\right\}$ is completely known up to a given cutoff length $\mathcal{L}$ allowing an exact evaluation of the periodic-orbit sum up to $\mathcal{L}$. Using Huber's law [9], Eq. (20),

$$
\frac{d N(l)}{d l} \sim \frac{e^{l}}{l}, l \rightarrow \infty,
$$

the remaining sum can be approximated by the following integral (see also [21]):

$$
\begin{aligned}
R_{1}(\mathcal{L}) \equiv & \frac{1}{2 \pi} \int_{\mathcal{L}}^{\infty} d l \frac{e^{l}}{l} \frac{\sin (p l)}{\sinh (l / 2)} \\
& \simeq \frac{1}{\pi} \operatorname{Im} \int_{\mathcal{L}}^{\infty} d l \frac{e^{[(1 / 2)+i p] l}}{l} \\
& \sim \frac{1}{\pi} \operatorname{Im} \int_{-[(1 / 2)+i p] \mathcal{L}}^{-i \infty} d t \frac{e^{-t}}{t} .
\end{aligned}
$$

The following integral over the integration path $\mathcal{C}_{R}$ along the quarter circle from $t=-i R$ to $R$ parametrized by $t=R e^{i \phi}, \phi \in\left[\frac{3}{2} \pi, 2 \pi\right]$, yields

$$
\begin{aligned}
\operatorname{Im} \int_{\mathfrak{C}_{R}} d t \frac{e^{-t}}{t} & =\int_{0}^{\pi / 2} d x e^{-R \cos x} \cos (R \sin x) \\
& =-\operatorname{si}(R)
\end{aligned}
$$

which vanishes for $R \rightarrow \infty$ because of $\lim _{R \rightarrow \infty} \operatorname{si}(R)=0$. Thus one can add

$$
\frac{1}{\pi} \operatorname{Im} \int_{\mathcal{C}_{R}} d t \frac{e^{-t}}{t}+\frac{1}{\pi} \operatorname{si}(R)=0
$$

to the last integral in (26). In the limit $R \rightarrow \infty$ one can connect the two integration paths and arrive at the integral representation for $\mathrm{E}_{1}(z)$,

$$
\mathrm{E}_{1}(z)=\int_{z}^{\infty} d t \frac{e^{-t}}{t}
$$

where it is assumed that the path of integration excludes the origin and does not cross the negative real axis. We are thus lead to $(p>0)$

$$
R_{1}(\mathcal{L}) \simeq \frac{1}{\pi} \operatorname{ImE}_{1}\left(-\left(\frac{1}{2}+i p\right) \mathcal{L}\right)
$$

and 


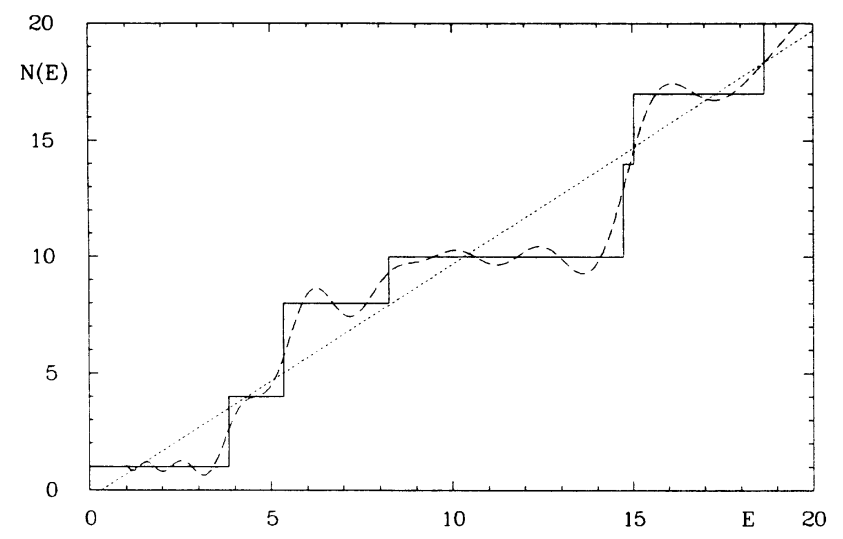

FIG. 2. The spectral staircase $\mathcal{N}(E)$ (solid curve) is shown for the regular octagon together with the curve obtained from (31) (dashed curve) for $\mathcal{L}=18.092$. Weyl's law $\mathcal{N}(E) \sim E-\frac{1}{3}$ is displayed as the dotted line.

$$
\begin{aligned}
\mathcal{N}(E) \simeq & 2 \int_{0}^{p} d p^{\prime} p^{\prime} \tanh \left(\pi p^{\prime}\right) \\
& +\frac{1}{2 \pi} \sum_{\left\{l_{n}\right\}} \sum_{\substack{k=1 \\
k l_{n} \leq \mathcal{L}}}^{\infty} \frac{\sin \left(p k l_{n}\right)}{k \sinh \left(k l_{n} / 2\right)} \\
& +\frac{1}{\pi} \operatorname{ImE}_{1}\left(-\left(\frac{1}{2}+i p\right) \mathcal{L}\right) .
\end{aligned}
$$

The first term in Eq. (31) gives for $E \rightarrow \infty$

$2 \int_{0}^{p} d p^{\prime} p^{\prime} \tanh \left(\pi p^{\prime}\right)=E-\frac{1}{3}+O\left(\sqrt{E} e^{-2 \pi \sqrt{E}}\right)$,

which is (apart from the exponentially small term) identi- cal to Weyl's law. In Figs. 2 and 3 we show $\mathcal{N}(E)$ for the regular octagon in the energy range $E \leq 20$ and $E \leq 200$, respectively. The dotted line corresponds to Weyl's law and is seen to describe the average behavior of $\mathcal{N}(E)$ reasonably well. The second and third terms in Eq. (31) are oscillating functions. For $\sqrt{E} \mathcal{L} \rightarrow \infty$, the third term behaves asymptotically as

$$
\begin{aligned}
\frac{1}{\pi} \operatorname{ImE}_{1}\left(-\left(\frac{1}{2}+i p\right) \mathcal{L}\right) \sim & \frac{1}{\pi E} \frac{e^{\mathcal{L} / 2}}{\mathcal{L}} \\
& \times\left[p \cos (p \mathcal{L})-\frac{1}{2} \sin (p \mathcal{L})\right] .
\end{aligned}
$$

Thus the energy resolution of the approximate formula (31) is determined by the cutoff length $\mathcal{L}$, $\Delta E \sim 2 \pi \sqrt{E} / \mathcal{L}$, and is getting worse with increasing energy for a fixed value of $\mathcal{L}$. In Figs. 2 and 3 we display as the dashed curve the evaluation of relation (31) for $\mathcal{L}=18.092$..., which means that more than 4000000 periodic orbits have been taken into account in the periodic-orbit sum in (31). One observes fluctuations around the staircase similar to the classical Gibbs phenomenon, and again the limited "input data" lead to a smoothing.

Although formula (31) is not able to resolve the higher-energy levels, it is seen from Fig. 3 that it gives an important improvement of Weyl's law. In our evaluation we have used the rest term in Eq. (31) in its form given there, but we have checked that the asymptotic formula (33) yields a sufficiently good approximation already for $E>6$. Notice that this rest term is large in the energy range considered, and that the good overall agreement seen in Figs. 2 and 3 would have been completely spoiled

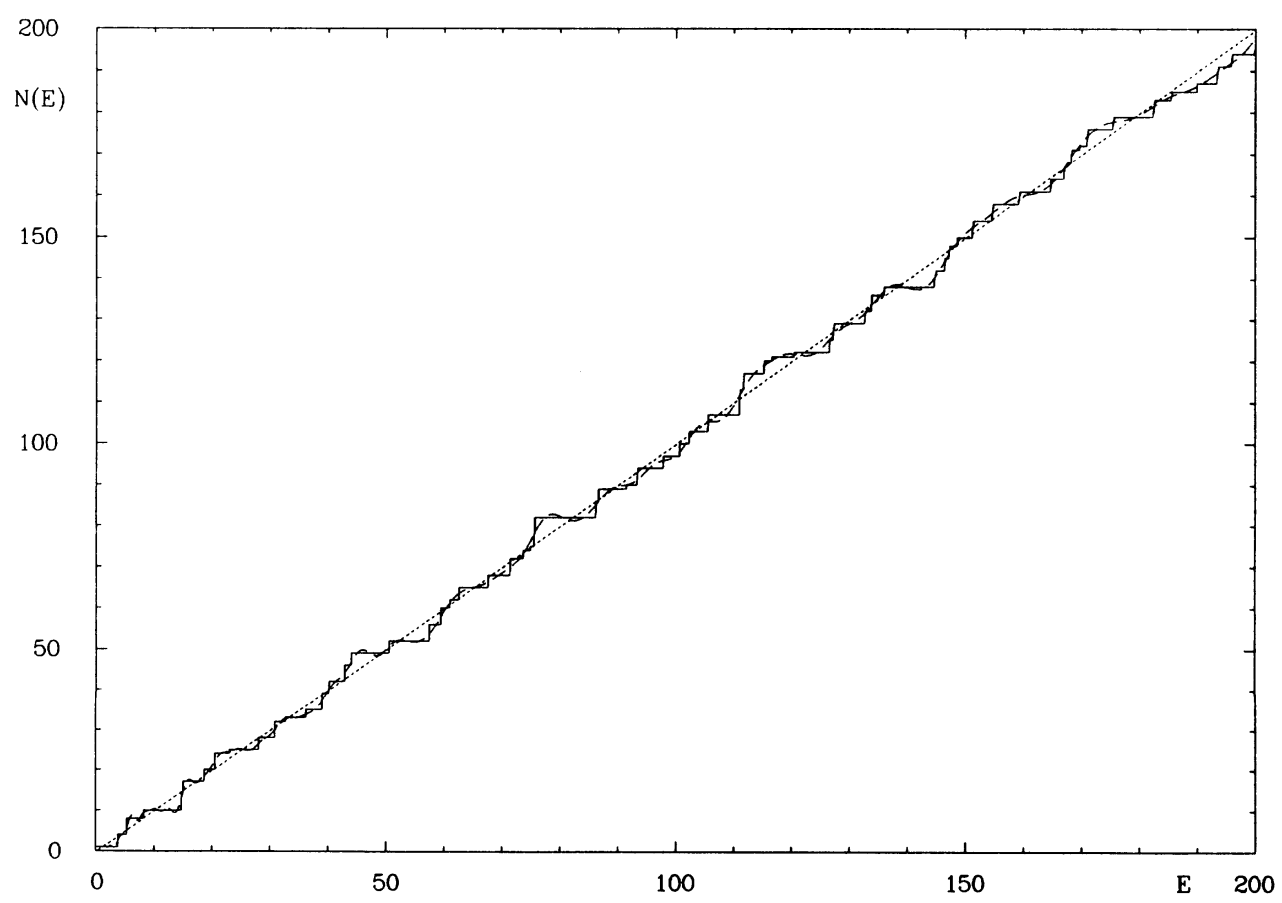

FIG. 3. The same curves as in Fig. 2 are shown but in the larger energy range $E \in[0,200]$. 
if this rest term would have been omitted. While the rest term diverges for $\mathcal{L} \rightarrow \infty$, it decreases for fixed $\mathcal{L}$ like $1 / \sqrt{E}$ in the semiclassical limit $E \rightarrow \infty$.

\section{A RULE FOR QUANTIZING CHAOS}

Recently, several authors [21-26] have proposed and/or numerically investigated various quantization rules for chaotic systems. In all these papers the quantization rules make essential use of the relevant dynamical $\zeta$ functions, which in the case of the octagons considered in the present paper and in the case of Artin's billiard are identical to the Selberg $\zeta$ function $[21,25]$.

Here we would like to propose a new rule for quantizing chaos, which is not based on the dynamical $\zeta$ function and thus does not require the validity of a functional equation as in the previous papers. The new quantization rule applies to chaotic systems in general, but here we will only illustrate it in the case of the octagons.

Having derived the approximation (31) for the spectral staircase $\mathcal{N}(E)$, it is natural to define approximate quantal energies $\widetilde{E}_{n}$ as solutions of the equation

$$
\widetilde{\mathcal{N}}\left(\widetilde{E}_{n}\right)=n+\frac{1}{2}, \quad n=0,1,2, \ldots,
$$

or, equivalently, as zeros of the function

$$
\xi_{1}(E) \equiv \cos [\pi \tilde{\mathcal{N}}(E)],
$$

where $\tilde{\mathcal{N}}(E)$ denotes the expression on the rhs of Eq. (31). A look at Figs. 2 and 3 suggests that the quantization condition (34) should yield reasonable approximations in those energy regions where the function $\widetilde{\mathcal{N}}(E)$ (the dashed curve in Figs. 2 and 3) does not show too large oscillations.

Table I gives the first quantal energies $\widetilde{E}_{n}, 1 \leq n \leq 19$, of the regular octagon, obtained from the quantization rule (34) in comparison with the quantal energies obtained by solving the Schrödinger equation directly using the method of finite elements (see Ref. [7]). (Only in the case where $n=8$ is the solution not unique, and the value $\widetilde{E}_{8}=7.85$ has been selected as the obvious one; see Fig. 2.) The table shows that the quantization condition (34) yields indeed reasonable approximations to the quantal energies. As has been discussed in detail in our earlier papers $[7,10]$, the regular octagon possesses many symmetries that are reflected by the twofold, threefold, and fourfold degeneracies, respectively, of the quantal energies, seen in the table and in Figs. 2 and 3. These degeneracies constitute a serious difficulty for any quantization condition. In fact, this was one of the reasons why the Riemann-Siegel look-alike formula [22], which has been tested in Fig. 6(a) of Ref. [21], was not able to generate even the first nine energies. It is seen from the table that the mean values $\left\langle\widetilde{E}_{n}\right\rangle$ calculated from the approximate eigenvalues $\widetilde{E}_{n}$ for those $n$-values for which the true eigenvalues are degenerate are excellent approximations to the true eigenvalues $E_{n}$, the relative error not larger than $1.5 \%$. We thus conclude that the quantization condition (34) works even in the very delicate situation of a system possessing high symmetries.

As a second test of the quantization condition (34), we
TABLE I. The first 19 quantal energies $E_{n}>0$ of the regular octagon. $E_{n}$ denotes the "true" eigenvalues taken from [7], whereas $\widetilde{E}_{n}$ denotes the approximations obtained from the quantization rule (34). $\left\langle\widetilde{E}_{n}\right\rangle$ is defined in the text.

\begin{tabular}{lccc}
\hline$E_{n}$ & $\widetilde{E}_{n}$ & $\left\langle\widetilde{E}_{n}\right\rangle$ & Relative error $(\%)$ \\
\hline 3.839 & 3.56 & 3.83 & -0.3 \\
3.839 & 3.81 & 3.83 & \\
3.839 & 4.11 & 3.83 & \\
& & & \\
5.354 & 5.01 & 5.40 & \\
5.354 & 5.31 & 5.40 & \\
5.354 & 5.52 & 5.40 & \\
5.354 & 5.74 & 5.40 & \\
& & & \\
8.250 & 7.85 & 8.13 & \\
8.250 & 8.40 & 8.13 & \\
& & & \\
14.726 & 14.24 & 14.55 & \\
14.726 & 14.47 & 14.55 & \\
14.726 & 14.66 & 14.55 & \\
14.726 & 14.84 & 14.55 & \\
& & & \\
15.049 & 15.02 & 15.25 & \\
15.049 & 15.23 & 15.25 & \\
15.049 & 15.49 & 15.25 & \\
18.659 & 18.15 & 18.74 & \\
18.659 & 18.76 & 18.74 & \\
18.659 & 19.32 & 18.74 & \\
\hline \hline
\end{tabular}

consider an asymmetric octagon that was already discussed in our earlier papers [8,21], and which has the nice property that near degeneracies are avoided between the first seven energy levels $\left(E_{n+1}-E_{n}>0.6\right.$ for $\left.n<7\right)$. In Fig. 6(b) of Ref. [21] we have shown an evaluation of the Riemann-Siegel look-alike formula for this octagon for $E \leq 6$. As in the case of the regular octagon, the result was not satisfactory, since only two of six eigenvalues were produced. In Fig. 4 we show an evaluation of the function $\xi_{1}(E)$, Eq. (35), for $E \leq 14$, using for the cutoff

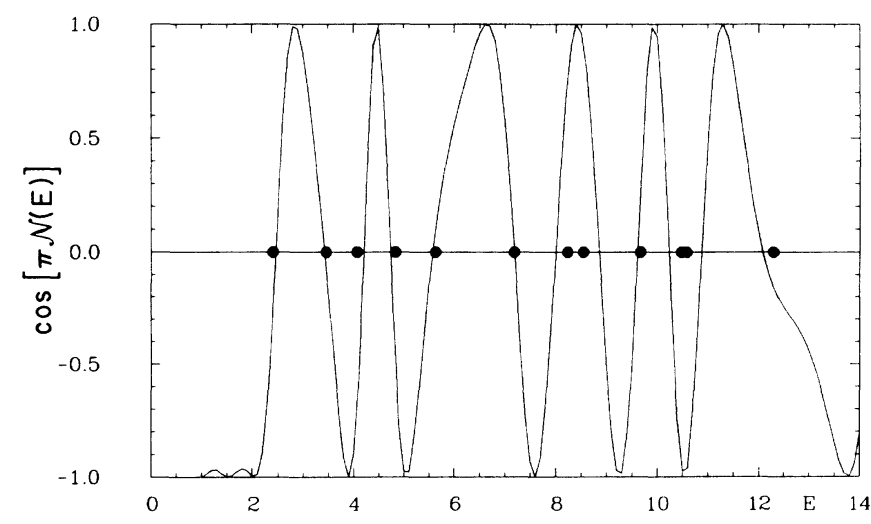

FIG. 4. For an asymmetric octagon $\cos [\pi \mathcal{N}(E)]$ is shown, where $\mathcal{N}(E)$ is computed by (31) with $\mathcal{L}=11.96242 \ldots$. The locations of the quantal energies computed by the method of finite elements are marked by bold dots. 
length $\mathcal{L}$ in Eq. (31) $\mathcal{L}=11.96242 \ldots$. (For this octagon the length spectrum is not completely known for $l_{n} \leq \mathcal{L}$, but a comparison of the computed length spectrum with Huber's law (20) indicates that only a few periodic orbits are missing. The length spectrum has been computed by using the algorithm described in [6], where all "words" consisting of maximal 12 "letters" have been considered.) The "true" quantal energies computed by the method of finite elements are shown as bold dots in Fig. 4. It is seen that the zeros of the function $\xi_{1}(E)$ indeed constitute good approximations to the true eigenvalues. Only in cases where two eigenvalues are very close to each other do the zeros of $\xi_{1}(E)$ show a tendency to be separated by a somewhat larger amount; see, for example, near $E=10.5$. But since the energy resolution is proportional to $1 / \mathcal{L}$, it is to be expected that the energy resolution would improve if a length spectrum could be used having a larger cutoff $\mathcal{L}$.

\section{THE SPECTRAL RIGIDITY $\Delta_{3}(L)$ FOR A TOY MODEL}

Apart from the level-spacing statistics, the spectral rigidity $\Delta_{3}(L)$ introduced by Dyson and Mehta [27] has played a major role in the study of statistical properties of quantal energy spectra for systems whose classical counterparts are chaotic. The statistics of these systems seem to be in good agreement with the predictions of random-matrix theory. The spectral rigidity is defined [27] as the average of the mean-square deviation of the staircase $\mathcal{N}(E)$ from the best-fitting straight line $a+b \varepsilon$ :

$\Delta_{3}(L) \equiv\left\langle\min _{(a, b)} \frac{1}{L} \int_{-L / 2}^{L / 2} d \varepsilon[\mathcal{N}(E+\varepsilon)-a-b \varepsilon]^{2}\right\rangle$,

where \langle\rangle denotes a local average. The constants $a$ and $b$ can be eliminated, yielding the well-known expression

$$
\begin{aligned}
\Delta_{3}(L)= & \left\langle\frac{1}{L} \int_{-L / 2}^{L / 2} d \varepsilon \mathcal{N}^{2}(E+\varepsilon)\right. \\
& -\left[\frac{1}{L} \int_{-L / 2}^{L / 2} d \varepsilon \mathcal{N}(E+\varepsilon)\right]^{2} \\
& \left.-12\left[\frac{1}{L^{2}} \int_{-L / 2}^{L / 2} d \varepsilon \varepsilon \mathcal{N}(E+\varepsilon)\right]^{2}\right\rangle .
\end{aligned}
$$

In [28] Berry derived a semiclassical theory for $\Delta_{3}(L)$ for integrable as well as for chaotic systems. This theory predicts a saturation of $\Delta_{3}(L)$ for $L \gg L_{\max }$ and a behavior consistent with random-matrix theory below $L_{\max }$.

We have already discussed in [8] several statistics computed directly from the quantal energies for 40 different octagons. Here we want to test numerically whether the approximation to $\mathcal{N}(E)$ computed by (31) is suited for a computation of $\Delta_{3}(L)$. As shown in Sec. III, this approximation gives a smoothed version of the "true" staircase and thus it is not obvious that one will obtain reasonable results for a statistics measuring discontinuous deviations from a best-fitting straight line.

To settle the question of what distinguishes the spectral rigidity $\Delta_{3}(L)$ of the true staircase from the smoothed version, we first investigate a toy model spectrum that can be treated analytically. Consider the energy spectrum $E_{n} \equiv n+\frac{1}{2}, n=0,1,2, \ldots$ having the same leading term in Weyl's law as the spectra of our Riemann surfaces. The staircase $\mathcal{N}(E)$ can then be expanded in a Fourier series

$$
\mathcal{N}(E)=E+\frac{1}{\pi} \sum_{n=1}^{\infty} \frac{(-1)^{n}}{n} \sin (2 \pi n E), \quad E \geq 0 .
$$

If the Fourier series is truncated at $N$, a smoothed version of the true staircase $\mathcal{N}(E)$ is obtained in analogy to the staircase of Sec. III. Inserting (38) into (37), all operations can be carried out, leading, after a tedious but straightforward computation, to

$$
\begin{aligned}
& \Delta_{3}^{N}(L)=\frac{1}{2 \pi^{2}} \sum_{n=1}^{N} \frac{1}{n^{2}} \\
& -\frac{1}{2 \pi^{4} L^{2}} \sum_{n=1}^{N} \frac{1}{n^{4}}\left[\sin ^{2}(\pi n L)\right. \\
& +3 \int \frac{\sin (\pi n L)}{\pi n L} \\
& \left.-\left.\cos (\pi n L)\right|^{2}\right] \text {, }
\end{aligned}
$$

with $\lim _{N \rightarrow \infty} \Delta_{3}^{N}(L)=\Delta_{3}(L)$.

In the limit $L \rightarrow \infty$ the second series in (39) vanishes, and one obtains the following exact expressions for the saturation values $\Delta_{\infty}^{N}$ and $\Delta_{\infty}$, respectively:

$$
\Delta_{\infty}^{N}=\frac{1}{2 \pi^{2}} \sum_{n=1}^{N} \frac{1}{n^{2}}, \Delta_{\infty}=\frac{\zeta(2)}{2 \pi^{2}}=\frac{1}{12} .
$$

Thus one immediately recognizes that the spectral rigidity $\Delta_{3}^{N}(L)$, i.e., the $N$ th approximation to the true staircase, saturates as expected, but at a value that is smaller than the true saturation value $\Delta_{\infty}$ by a constant amount given by

$$
C^{N} \equiv \frac{1}{12}-\frac{1}{2 \pi^{2}} \sum_{n=1}^{N} \frac{1}{n^{2}} .
$$

In the opposite limit $L \rightarrow 0$, the approximation (39) can be expanded in $L$, yielding (for fixed $N$ )

$$
\Delta_{3}^{N}(L) \sim \frac{\pi^{2}}{90} \frac{N(N+1)(2 N+1)}{6} L^{4}, L \rightarrow 0 .
$$

This contrasts with the exact linear behavior $\Delta_{3}(L) \sim \frac{1}{15} L$, $L \rightarrow 0$. The difference is caused by the absence of discontinuities in the smoothed version of the staircase. If the limit $N \rightarrow \infty$ is carried out at fixed $L$, the coefficient of the $L^{4}$ term diverges. On the other hand, one sees that (42) behaves for large $N$ as $(N L)^{3} L$, which shows that the correct behavior for $L \rightarrow 0$ is obtained if one considers the "scaling limit" $N \rightarrow \infty, L \rightarrow 0$, but keeping $N L$ fixed. We thus obtain the approximation

$$
\Delta_{3}(L) \simeq \Delta_{3}^{N}(L)+C^{N}
$$

which should hold for $L \gg 1 / N$. 


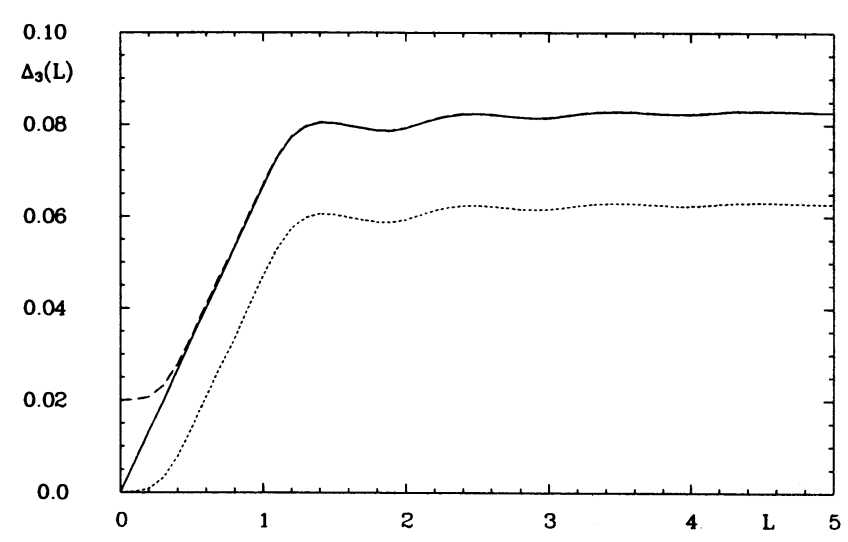

FIG. 5. The spectral rigidity $\Delta_{3}(L)$ (solid curve) for the toy model spectrum $E_{n}=n+\frac{1}{2}$ is shown in comparison with the approximation (39) for $N=2$ (dotted curve). The dashed curve is obtained by shifting the dotted curve by the constant $C^{2}$ given in $(41)$.

In Fig. 5 the spectral rigidity $\Delta_{3}(L)$ is shown in comparison with the second approximation $[N=2$ in (39)], which is too low by a constant amount $C^{2}=0.020$. . as derived from Eq. (41). Shifting by this constant corrects not only the saturation value, but also leads to a very good description of $\Delta_{3}(L)$ for $L>\frac{1}{2}$. Only for $L<\frac{1}{2}$ does the mentioned vanishing proportional to $L^{4}$ cause an error in the description of $\Delta_{3}(L)$. In summary, one sees that a truncation of the Fourier series (38) leads only to a constant shift of the spectral rigidity if one excludes a small region near $L=0$.

\section{THE SPECTRAL RIGIDITY $\Delta_{3}(L)$ FOR TWO STRONGLY CHAOTIC SYSTEMS}

After the discussion of the toy model presented in the last section, let us now turn to the computation of the spectral rigidity for two strongly chaotic systems defined by the two octagons already considered in the previous sections. Inserting the approximation $(31)$ for $\mathcal{N}(E)$ into the general expression (37), we obtain a (very long) formula, which will not be reproduced here, and which expresses the rigidity in terms of the classical periodic orbits. Since the sum over the length spectrum has to be cut off at a maximal length $\mathcal{L}$, one cannot expect that the resulting rigidity exactly reproduces the true rigidity calculated directly from the quantal energies. Rather, one expects, as in the case of the toy model [see Eq. (43)], that for large $L$ values the rigidity is shifted by a constant that depends only on the cutoff $\mathcal{L}$.

The numerical evaluation of $\Delta_{3}(L)$ is shown in Fig. 6(a) for the regular octagon (using $\mathcal{L}=18.092$ ) and in Fig. 6(b) for the asymmetric octagon already discussed in Sec. IV (using $\mathcal{L}=11.96242$ ). The solid curves represent the "true" rigidity computed directly from the quantal energies, while the open circles are the approximations obtained from Eq. (31). The larger values obtained for $\Delta_{3}(L)$ in the case of the regular octagon in comparison with the asymmetric octagon are caused by the high symmetry the regular octagon possesses. Indeed, up to
$E=200$ there are only a few quantal energies that are not degenerate and thus the spectral staircase $\mathcal{N}(E)$ has exceedingly large steps, as seen in Fig. 2 and 3. On the other hand, the asymmetric octagon has only parity symmetry, and its spectrum should therefore behave as a superposition of two independent GOE spectra [dashed curve in Fig. 6(b) ] according to the random-matrix theory (GOE refers to the Gaussian orthogonal ensemble of random matrices). As expected, the approximation (31) yields for $\Delta_{3}(L)$ values that lie below the true rigidity. But shifting the open circles in Figs. 6(a) and 6(b) by a constant (1.004 in the case of the regular octagon; 0.385 for the asymmetric octagon), one observes that the result represented by the closed circles agrees nicely with the true rigidity for $10<L \leq 150$ (regular octagon) and $50<L \leq 150$ (asymmetric octagon), respectively. For the latter system, one gets agreement with the true curve within $10 \%$ in the full range $L>15$. As in the case of the toy model, the approximation fails in a region near $L=0$, which is caused by the fact that the sharp discontinuities in the spectral staircase are smoothed. For both systems,
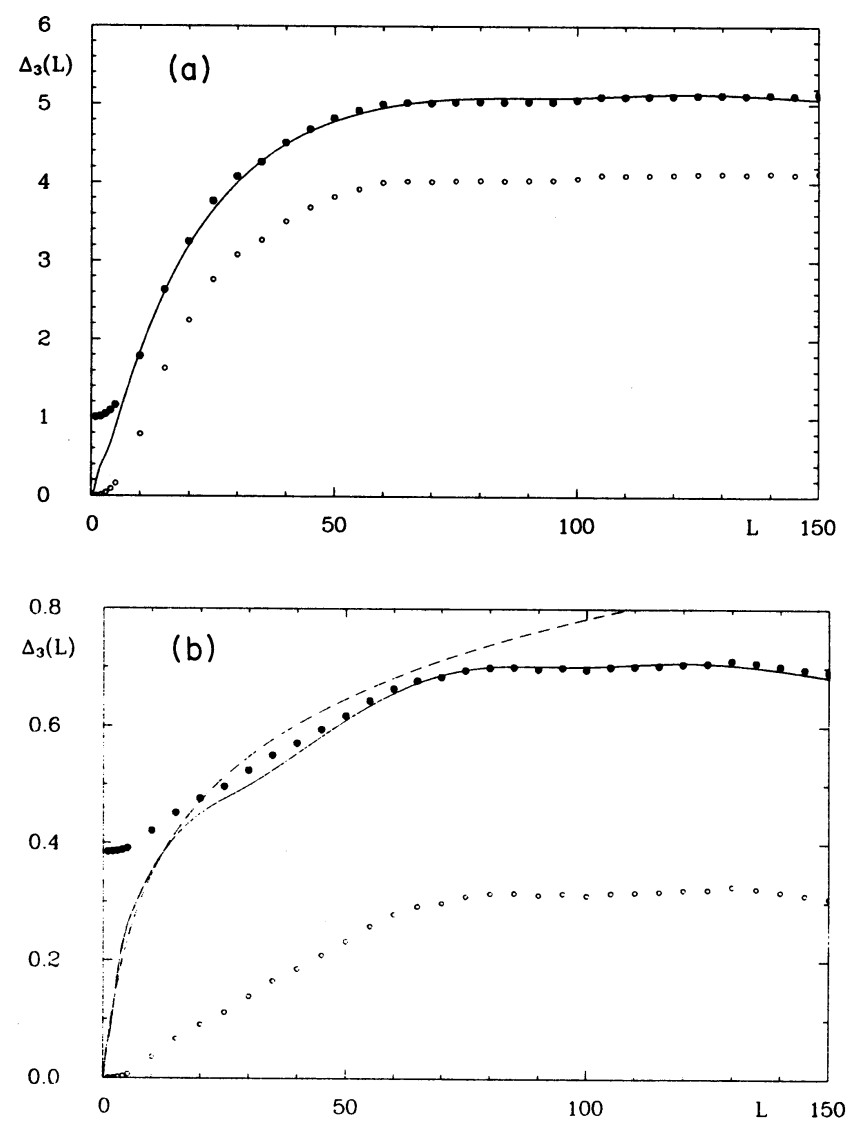

FIG. 6. The solid curves represent $\Delta_{3}(L)$ obtained directly from the quantal energy spectrum up to $E=200$ for the regular octagon (a) and for the asymmetric octagon (b). The open circles correspond to the approximation (31). The full circles represent the theoretical prediction after adding a constant, as discussed in the text. The dashed curve in (b) shows the prediction of random-matrix theory for the superposition of two independent GOE spectra. 
the rigidity saturates nonuniversally at a finite value $\Delta_{\infty}$ for $L>70$, in agreement with the semiclassical theory of Berry [28]. Notice, however, that the theory presented in [28] does not apply directly to the present case, since the two octagons have not been desymmetrized. There remains the challenge to develop for the systems considered here a theory that expresses the saturation value $\Delta_{\infty}$ completely in terms of the periodic orbits. What is required is a formula analogous to Eq. (41) for the constant shift in dependence of the cutoff $\mathcal{L}$.

\section{DISCUSSION}

In this paper we have discussed several applications of the Selberg trace formula, Eq. (1), which has for the Hadamard-Gutzwiller model considered here exactly the same form as Gutzwiller's semiclassical periodic-orbit theory [1-3]. It is this analogy between Gutzwiller's and Selberg's trace formulas that makes the motion on compact Riemann surfaces so interesting and gives these particular chaotic systems the unique position as prototype models for the study of quantum chaology. We anticipate that the results of this paper can be easily generalized to analogous semiclassical periodic-orbit formulas for general chaotic systems.

Starting from the sum rule (4) for the trace of the cosine-modulated heat kernel, we derived in Sec. II the explicit formulas (8) and (10) for the classical staircase $N(l)$, which can be considered in analogy with Riemann's prime-number formulas (14) and (15) as "generalized prime-number formulas" for the "generalized primes" defined by $e^{l_{n}}$. These formulas are interesting because they show explicitly the striking duality that exists between the lengths of the classical periodic orbits and the quantum-mechanical spectrum. From these formulas we were able to derive immediately not only Huber's law (20), which plays the role of a "generalized prime-number theorem" in analogy with the famous prime-number theorem (17), but also an explicit formula for the remainder function $Q(l)$ defined by

$$
N(l)=\operatorname{Ei}(l)+Q(l) .
$$

By approximating $Q(l)$ by the first 200 quantal energies, we obtained for the regular octagon the result shown in Fig. 1, which reproduces nicely the main features of the periodic-orbit staircase function in the short-length limit, illustrating explicitly how the fine structure of the classical periodic orbits is determined by the quantal energies. This approach, which we have called "inverse quantum chaology," can be characterized by the question "Can one hear the periodic orbits of a compact Riemann surface?", a variation of the famous question posed by Kac [29].

Asymptotically, the remainder $Q(l)$ is given by [see Eq. (22)]

$Q(l)=\frac{e^{l / 2}}{l}\left[2 \sum_{n=1}^{\infty} \frac{\cos \left(p_{n} l-\alpha_{n}\right)}{\sqrt{E_{n}}}-1\right]+\cdots$.

Since $E_{n} \sim n\left(p_{n} \sim \sqrt{n}, \alpha_{n} \rightarrow \pi / 2\right)$ for $n \rightarrow \infty$ according to Weyl's law, there are obviously some serious questions about convergence here that we have not discussed in this paper. By analogy with the Riemann $\zeta$ function, one would expect that $Q(l)$ behaves as $Q(l)=O\left(e^{(1 / 2+\epsilon) l}\right)$, since the Riemann hypothesis is valid for the Selberg $\zeta$ function $Z(s)$ defined by the Euler product

$$
Z(s)=\prod_{\left\{l_{n}\right\}} \prod_{k=0}^{\infty}\left(1-e^{-(s+k) l_{n}}\right), \quad \operatorname{Res}>1 .
$$

[Notice that the nontrivial zeros of $Z(s)$ are related to the quantal energies via $s_{n}=\frac{1}{2} \pm i p_{n}$.] It is not difficult to obtain the estimate $Q(l)=O\left(e^{7 l / 8}\right)$, but yet the best result known today is only of the type $Q(l)=O\left(e^{3 l / 4} / l^{\alpha}\right)$ with $\alpha=\frac{1}{2}$ [30] and 1 [31]. (Here we assume again that there are no small eigenvalues.) We may add that our numerical results are consistent with $Q(l)=O\left(e^{l / 2} / l\right)$, but this is, of course, no proof.

In Sec. III we have studied the periodic-orbit formula (24) for the spectral staircase $\mathcal{N}(E)$. Although Eq. (24) is an exact relation that is valid in the sense of distributions, it is not obvious at all that this relation can be used for an actual numerical calculation. If the integral term in (24) having the meaning of a mean spectral staircase given asymptotically by Weyl's law, see Eq. (32), is denoted by $\overline{\mathcal{N}}(E)$, relation (24) is equivalent to the exact formula

$$
\mathcal{N}(E)=\overline{\mathcal{N}}(E)+\frac{1}{\pi} \arg Z\left[\frac{1}{2}+i p\right],
$$

where $Z\left[\frac{1}{2}+i p\right]$ means here the analytic continuation of the Selberg $\zeta$ function (46) to the critical line $\operatorname{Res}=\frac{1}{2}$. Thus our final equation (31) for $\mathcal{N}(E)$ is essentially an approximate formula for the phase of the Selberg $\zeta$ function on the critical line. It is crucial for this formula to be numerically meaningful that we have truncated the series over the periodic orbits at the cutoff $\mathcal{L}$ and that we have found a good estimate for the remainder; see Eq. (30). Notice that this remainder involves actually a "renormalization procedure" that has been carried out in the last step in (26). The numerical results presented in Figs. 2 and 3 seem to indicate that the approximation (31) is indeed meaningful. There arises again the difficult problem of finding a good estimate similarly as for the remainder $Q(l)$. If the last term in Eq. (47) is denoted by $S(p)$, our numerical calculations are consistent with $S(p)=O(1)$, which is far beyond the best estimate obtained rigorously up to now, which gives $S(p)=O(p / \ln p)$ [30]. (This estimate, as well as the estimate for $Q(l)$, was first provided by Selberg around 1950-52 in an unpublished manuscript; see, e.g., [32]. The result for $Q(l)$ with $\alpha=\frac{1}{2}$ was obtained independently by Huber [33].)

The rule for quantizing chaos proposed in Sec. IV relies on the continuous approximation (31) to the discontinuous spectral staircase $\mathcal{N}(E)$, which takes positive half-integer values if $E$ is close to a quantal energy. Thus the zeros of the function (35) should yield good estimates for the quantal energies. Our numerical evaluations presented in Table $I$ and Fig. 4 give strong support to this new rule. Based on the exact functional equation for the Selberg $\zeta$ function, we have recently proposed [21] a 
different quantization rule, which defines the quantal energies by the zeros of the function

$$
\xi(p) \equiv \operatorname{Re}\left[e^{i \pi \overline{\mathcal{N}}(E)} Z\left(\frac{1}{2}+i p\right)\right] .
$$

(For related quantization rules see also [22-26].) The evaluation of $Z(s)$ on the critical line using a truncated Dirichlet series did not provide useful results for $\xi(p)$ in the case of the octagons, which could be explained by the fact that the Dirichlet series was not even conditionally convergent on the critical line. (The quantization condition based on (48) was, however, very successful in the case of the hyperbola billiard [24] and of Artin's billiard [25], where the corresponding Dirichlet series shows a better behavior.) Thus it appears that the new rule for quantizing chaos is superior to the quantization condition based on (48), since it works even in cases where the Dirichlet series for $Z(s)$ diverges on the critical line.
Finally, in Sec. VI we have used the approximation (31) to calculate the spectral rigidity, which is an important measure of the fluctuations of the energy spectrum. The results presented in Figs. 6(a) and 6(b) show the expected saturation for large $L$. Adding a constant led to a good description of the rigidity in the whole range above $L=15$. Extrapolating from the toy model studies in Sec. $\mathrm{V}$, it is obvious that a calculation of the constant would require a resummation of the long orbits, which will be studied elsewhere.

\section{ACKNOWLEDGMENTS}

We would like to thank the Deutsche Forschungsgemeinschaft for financial support under contract number DFG-Ste 241/4-3 and the HLRZ at Jülich for the access to the CRAY Y-MP 832 computer.
[1] M. C. Gutzwiller, J. Math. Phys. 8, 1979 (1967); 10, 1004 (1969); 11, 1791 (1970); 12, 343 (1971).

[2] M. C. Gutzwiller, Chaos in Classical and Quantum Mechanics (Springer, New York, 1990).

[3] M. C. Gutzwiller, Phys. Rev. Lett. 45, 150 (1980); Phys. Scripta T 9, 184 (1985).

[4] A. Selberg, J. Indian Math. Soc. 20, 47 (1956).

[5] N. L. Balazs and A. Voros, Phys. Rep. 143, 109 (1986).

[6] R. Aurich and F. Steiner, Physica D 32, 451 (1988).

[7] R. Aurich and F. Steiner, Physica D 39, 169 (1989).

[8] R. Aurich and F. Steiner, Physica D 43, 155 (1990).

[9] H. Huber, Math. Ann. 138, 1 (1959).

[10] R. Aurich, E. B. Bogomolny, and F. Steiner, Physica D 48, 91 (1991).

[11] R. Aurich, M. Sieber, and F. Steiner, Phys. Rev. Lett. 61, 483 (1988).

[12] G. H. Hardy and E. M. Wright, An Introduction to the Theory of Numbers, 5th ed. (Oxford University, London, 1979).

[13] B. Riemann, Monatsber. Königl. Preuss. Akad. Wiss. Berlin (1859), p. 671; reprinted in B. Riemann, Gesammelte Mathematische Werke (Dover, New York, 1953), p. 145.

[14] H. von Mangoldt, J. Reine Angew. Math. 114, 255 (1895).

[15] J. Hadamard, Bull. Soc. Math. France 24, 199 (1896).

[16] C. J. de la Vallée Poussin, Ann. Soc. Sci. Bruxelles 20, 183 (1896).

[17] H. Riesel, Prime Numbers and Computer Methods for Fac- torization (Birkhäuser, Boston, 1985).

[18] J. E. Littlewood, C. R. Acad. Sci. Paris 158, 1869 (1914).

[19] D. Zagier, Math. Intelligencer, Vol. 0, August 1977, p. 7.

[20] H. Riesel and G. Göhl, Math. Comp. 24, 969 (1970).

[21] R. Aurich and F. Steiner, Proc. R. Soc. London, Ser. A (to be published).

[22] M. V. Berry and J. P. Keating, J. Phys. A 23, 4839 (1990); J. P. Keating, Proc. R. Soc. London, Ser. A (to be published).

[23] E. B. Bogomolny, Comments At. Mol. Phys. 25, 67 (1990); Nonlinearity (to be published).

[24] M. Sieber and F. Steiner, Phys. Rev. Lett. 67, 1941 (1991).

[25] C. Matthies and F. Steiner, Phys. Rev. A 44, 7877 (1991).

[26] G. Tanner et al. Phys. Rev. Lett. 67, 2410 (1991).

[27] F. J. Dyson and M. L. Mehta, J. Math. Phys. 4, 701 (1963).

[28] M. V. Berry, Proc. R. Soc. London; Ser. A 400, 229 (1985).

[29] M. Kac, Am. Math. Monthly 73, 1 (1966).

[30] D. A. Hejhal, The Selberg Trace Formula for PSL(2,R), Vol. I, Lecture Notes in Mathematics Vol. 548 (Springer, Berlin, 1976); The Selberg Trace Formula for PSL(2,R), Vol. II, Lecture Notes in Mathematics Vol. 1001 (Springer, Berlin, 1983).

[31] P. X. Gallagher, cited in H. Iwaniec, J. Reine Angew. Math 349, 136 (1984).

[32] A. Selberg, in Proceedings of the International Mathematical Congress, Stockholm.

[33] H. Huber, Math. Ann. 142, 385 (1961); 143, 463 (1961). 